\title{
The Research of Water level Control System Based on AT89S52
}

\author{
Yong Shi ${ }^{1, a}$, Xin Liü ${ }^{2, b}$, Wei Wang ${ }^{3, c}$, Ning Tao ${ }^{4, d}$ and Chong $\mathrm{Xu}^{5, \mathrm{e}}$ \\ ${ }^{1}$ BIM Computing Research Center, Shenyang jianzhu University, Shengyang, 110168, China \\ 2 BIM Computing Research Center, Shenyang jianzhu University, Shengyang, 110168, China \\ ${ }^{3}$ BIM Computing Research Center, Shenyang jianzhu University, Shengyang, 110168, China \\ ${ }^{4}$ BIM Computing Research Crnter, Shenyang jianzhu University, Shengyang, 110168, China \\ ${ }^{5}$ BIM Computing Research Crnter, Shenyang jianzhu University, Shengyang, 110168, China \\ a Shiyong13@163.com, b6620274@qq.com, ${ }^{\text {'5 } 519836646 @ q q . c o m, ~}$ \\ 23010150@qq.com, $32500103 @ q q . c o m$,
}

Keywords: Automatic control; Tank; Water level control

\begin{abstract}
AT89S52 microcontroller of the control system for the control of the core, combined with sensor technology, amulti control functions in one, including temperature, automatic lighting, automatic water level control, to achieve the control of fish tank.
\end{abstract}

\section{Introduction}

Currently, many families and hotels, shopping malls and other public places are still using an artificial hand for viewing the water tank test, level control, water cycle, feeding and other operations. For the aquarium's conservation issues, the market also has been found in a variety of fish tank water temperature control, drainage ${ }^{[1-3]}$, aeration and lighting equipment, such as filters, heaters, pumps and oxygen equipment to improve water quality. However, as to many products, function is not uniform, and mostly non-intelligent, single, constant temperature control, oxygenation, or lighting systems ${ }^{[4-6]}$. Ornamental fish tanks for manual operation bring a lot of inconvenience, thus the focus of this paper is to watch the fish tank on the current status of control equipment, analytical and research applications, a fish tank control system design ${ }^{[7,8]}$.

\section{Programs and Functions of the Overall System}

\section{System Functional Components}

The system was developed with the full knowledge and analysis of various types of aquarium controllers currently carried out under the premise of the whole system is divided into the following functional subsystems: the automatic heating / cooling temperature control, automatic / timed aeration system, automatic Water level control system, automatic lighting systems. These subsystems have their own input and control output signal detection capabilities, combined with the system clock circuits, data storage circuit / watchdog / reset circuit, and the function of each sub system parameter settings and the LCD display circuit, a common integration set of functional control system.

\section{System Control Parameters}

The control object for the tank system, the control aim is to make the system automatically adjust to providing optimum aquarium water quality and living environment. Note that the water quality design and environmental parameters: water temperature, dissolved oxygen of water, the water level is high or low ambient lighting and other parameters. Based on the same design principles and methods for other environmental requirements, different environmental parameters can be controlled to achieve a unified design to improve scalability. Table 1 shows the parameters of the control system processing.

The Hardware Structure Diagram and the Overall Structure. System's hardware The system microcontroller core CPU, the formation of a tank outside can be placed in stand-alone, separate 
Table 1 control parameters

\begin{tabular}{|c|c|c|}
\hline Project & Control parameters & Corresponding measures \\
\hline 1 & Water temperature & $\begin{array}{l}\text { According to the system settings control the heating temperature } \\
\text { range of bars to start, stop }\end{array}$ \\
\hline 2 & Dissolved Oxygen & $\begin{array}{l}\text { Oxygen content of oxygen under control pump start and stop- } \\
\text { Time to time or according to the system to start, stop }\end{array}$ \\
\hline 3 & Water level & Inlet valve under the control of water level start, stop, \\
\hline 4 & Lighting & $\begin{array}{l}\text { Set the time according to the system timing control lights turn on } \\
\text { and off }\end{array}$ \\
\hline
\end{tabular}

ways to achieve water quality and environmental testing signal input and output control signals from various quarters, the system hardware design shown in Fig. 1.

Central control module: The main microcontroller as the core, including the crystal oscillator, reset circuit, extended memory, and so on. Interactive button control module: the function keys for system settings, and set the appropriate course of LCD liquid crystal display control to achieve a good human-computer interaction function.

In real time through the LCD liquid crystal display parameter values.

Generated by LCD gauge tube and the buzzer sound and light alarm. Clock control circuit module: Implementing the system clock and the absolute control of the relative clock synchronization is time for environmental parameters The number of detection and the control device start and stop operation of the key. Data Storage / watchdog / reset the module: the achievement of key parameters of the storage, system working process of monitoring and restart the reset exception.

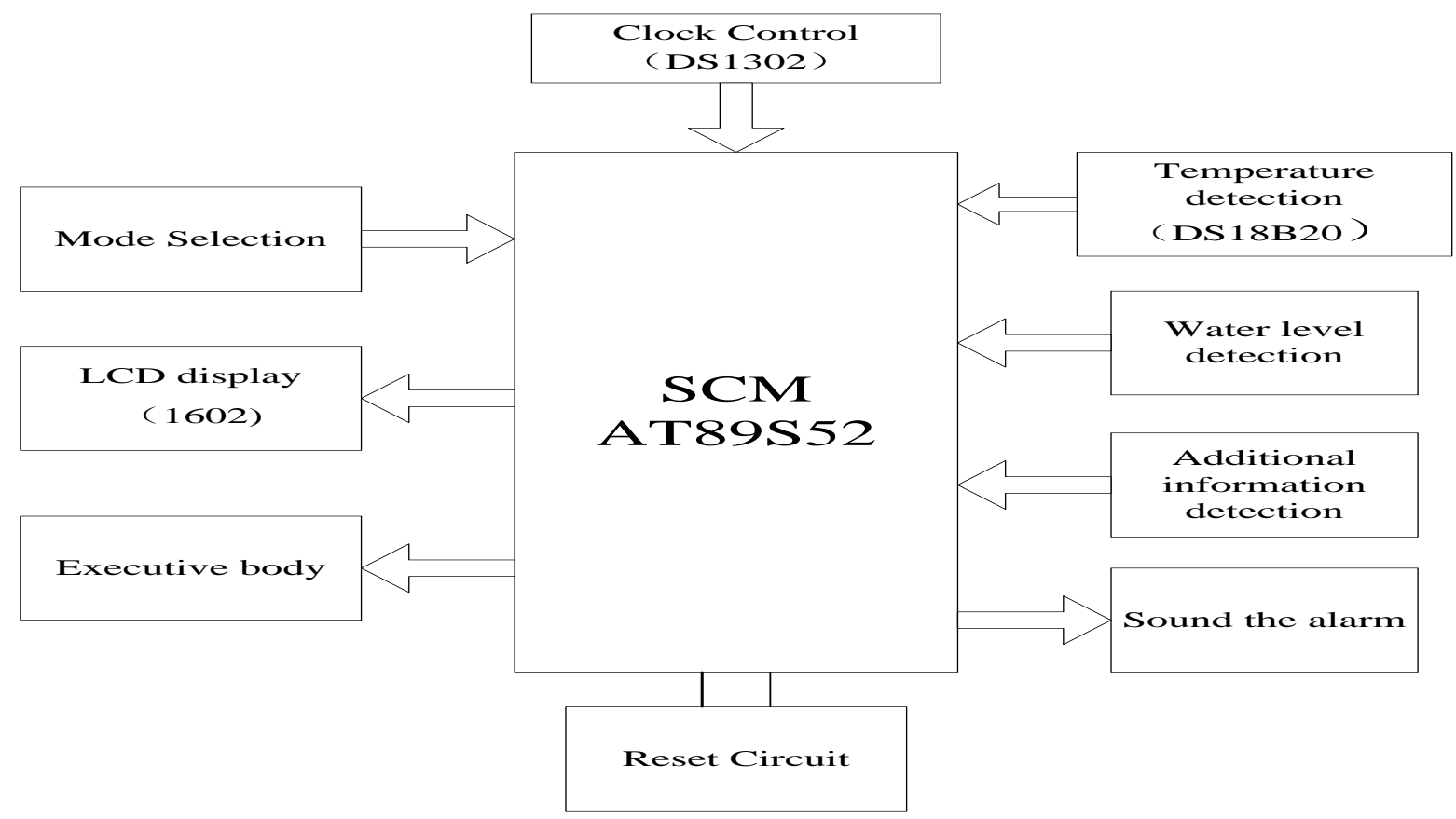

Figure 1. Finite System hardware block diagram

The overall structure of the hardware. Microcontroller hardware and software design are two important aspects of the system. The system hardware design, the main departure from the following principles:

Hardware design and software design optimized combination of hardware circuitry. Some of the hardware features are available for software to implement, in turn, the function of some of the software can also be used to complete the hardware. Hardware goes with the software to function. The software's response time is not only longer than hardware, but also takes CPU time. However, the function of the hardware with software is to simplify the hardware structure and improve the reliability of hardware, simplifying system upgrades, etc. It can also reduce costs, and therefore the sys- 
tem design process is in real time to meet the feasibility and possible under the premise Possible hardware functions implemented in software.

Reliability and anti-jamming design. According to the reliability design theory, the system is less than the number of chips used in the system longer MTBF, and the less number of chips used, the address data bus on the circuit board, the less likelihood of interference, so the basic system Microcontroller. The idea is to meet the function of the number of cases and strive to use fewer chips and circuits, the system selected DS18B20 digital temperature sensor is also based on this consideration. The system mostly used features advanced DIP devices, so from the component count, board space, power consumption, interference and system costs have been significantly improved.

Flexible and feature upgrades and system expansion. A design is often not fully taken into account all aspects of the system. The system needs to constantly improve, the need for functionality upgrade; and, the system should be considered in the design of applications in the future extension of the convenience. Extensions of the original design when the system should not need to conduct in case of big changes, modify the software and hardware, even a small amount can be done without modifying the hardware. Extensions are flexible in a measure of the importance of the merits of a system pointer. For example, the system can control a total of 8 outputs, the main control section takes up only 4 , if you need to increase multi-channel control, system hardware extension is very convenient, and only minor modifications to the software can be completed. According to the system hardware requirements and some design principles above, determine the system hardware schematic diagram. AT89S52 chip microcomputer system for the central processing unit, the temperature sensing lement, water level sensors, liquid crystal display, sound and light

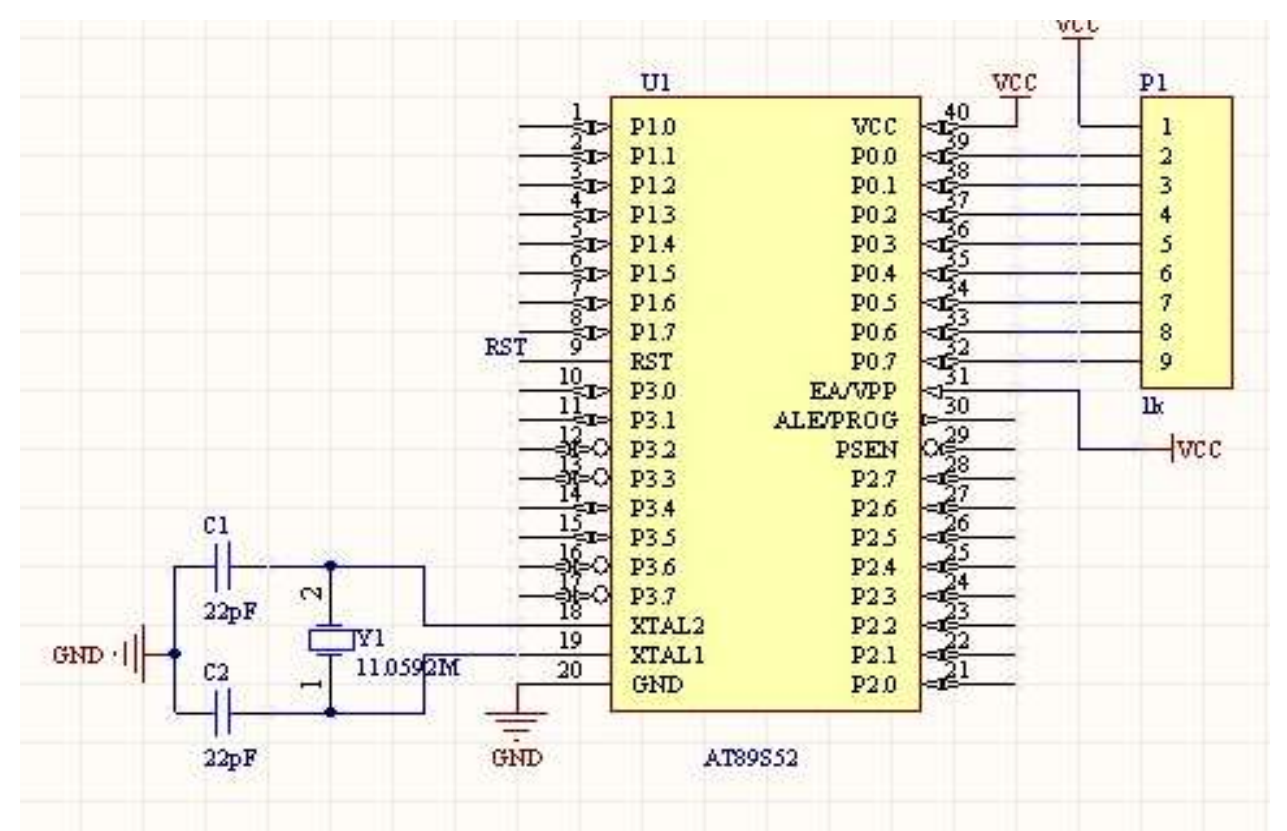

Figure 2. Finite The host controller hardware circuit diagram

\section{Host Controller Hardware Design}

As the core to form the main controller of AT89S52 the hardware circuit, shown in Fig. 2. Hardware components:

Real-time clock circuit: the 7,6,5 feet from the DS1302 and the CPU of the P2.4, P2.5, P2.3 connected to form;

Reset circuit: the key S4 the RST pin CPU connection with the composition;

Keyboard interface circuit: S1, S2, S3 button pin and CPU of theP1.0 P1.2 connected to form;

LCD 1602 interface circuit: DB0 DB7 and the CPU of the P0.0 P0.7, RS, R / W, E and P2.2, P2.1, P2.0 connected to form; 
Temperature detection circuit: the DS18B20 the I / O pins and the CPU's P2.6 connected to form; Buzzer circuit: and the CPU's P2.7 connected to form;

Actuator drive circuit: the relay and the CPU of the P3.0, P3.1, P3.2 connected to form.

\section{Software Design}

Control system software design is the design of the tank's main content and the focus of the system should be based on functional requirements to the system hardware as the basis for system software design. To make software features designed specifically to read and convenient debugging, reliability, and general use of structured programming. SCM SCM system program is used to develop the C programming language, the SCM system, the program is divided into six modules, namely the main program module, time and temperature acquisition module, detection module collection tank environmental parameters, keyboard control and display processing module, and Data storage, power protection, power on reset and watchdog module. Each module has certain features, some of which also contain a number of sub-modules, both independent and interconnected, low-level module can be called high- level module. System software described in this article includes: the main program, initialization routines, interrupt service routine, sampling and testing procedures, keyboard and display processing, sampling and testing procedures, state control procedures, and data storage, powerdown protection, Power-on reset and watchdog program. The following description of each of the program modules will be the design and preparation. System main program flow chart is shown in Fig. 3. Main program code is as follows:

void delay (unsigned int delay_i); / / delay procedures

void display1 ();// display program

void Init ();// initialize

void chuli1302 ();// time handler

void an jian ();// function keys

void wendu18b20 ();// function of temperature treatment

void xian wei kai guan ();// motor control function electric bar lamp

void main ()

\{

Init ();// initialize including the $130218 \mathrm{~b} 201602$ int1 initialization function, etc.

while (1)

\{

xian wei kai guan ();// motor control function electric bar lamp

an jian ();// function keys

chuli1302 ();// time handler

wendu18b20 ();// function of temperature treatment

display1 (); / / display program

\} 


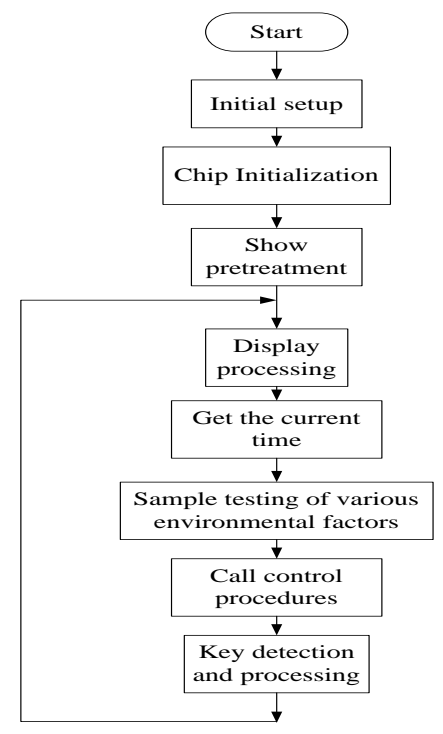

Figure 3. Finite System main program flow chart

\section{Conclusion}

The results show that through the practice of the control system stable and reliable, easy operation, while the system is designed to be flexible, simple, low cost, which has some use and reference.

\section{References}

[1] Xuehai Hu. Microcontrol ler theory and applications design. Beijing: Electronic Industry Press, 2005:3 6

[2] Tao chen. microcontroller applications and C51 programming. Beijing: Mechanical Industry Press, 2008:6 18.

[3] Yong quan yu World popular MCU Technical Manual -U.S.

Series Beijing: BeijingAeronautics and Astronautics Press 2004.pp.3-5.

[4] Xin Zhang. microcontroller theory and applications. Beijing: Electronic Industry Press, 2005:6 13.

[5] Atmel corporationat.8-bit Microcontroller with 8K Bytes In-System Programmable Flash AT89S52. www.atmel.com/atmel/acrobat/doc1919.pdf, 2010.5.29.

[6] Hailan Zhao, Jian ZHU, Xiangwei Zhao. DS1302 real-time display of time and application of the principle. Electronics. $2004:$ pp.43-46.

[7] HUANG Zhi. start with the real time clock chip DS1302 data acquisition system. microcontroller and embedded system applications .2003, (1-6): pp. 365-367.

[8] jiacai fu. SCM control engineering practice technology. Beijing: Chemical Industry Press, 2004:pp.155 -160. 